\title{
Editorials
}

\section{Somatosensory evoked potentials during spinal surgery}

the clinical intraoperative utility of SSEP have been topics of debate since the introduction of this technique into the operating room more than twenty years ago. The intraoperative use of evoked potentials appears to be increasing in North America, stimulated in part by the position paper put forth by the Scoliosis Research Society in 1992 which endorsed the routine use of SSEP during scoliosis instrumentation surgery for preventing spinal cord injury. ${ }^{2}$ Whether it is the litigious climate we find ourselves in or the belief that SSEP monitoring will truly benefit patients undergoing spinal surgery SSEPs will likely become a common component of OR monitoring equipment in the foreseeable future.

The intraoperative use of SSEP is not limited to spinal surgery as it is also used as a neuromonitor in carotid artery, neurovascular, acetabular and surgery involving aortic cross-clamping. Although SSEP monitoring may also be useful during intra- and extracranial vascular surgery, acetabular procedures and surgery involving aortic cross-clamping, this discussion is restricted to the use of SSEP monitoring during spinal surgery.

Several important questions must be answered. Can SSEP be reliably recorded in the OR without placing the patient at an increased risk of morbidity directly related to the monitoring? Do intraoperative SSEP changes correlate with postoperative neural deficits? Can intraoperative SSEP changes be used to prevent neural injury from occurring? Do the benefits warrant the additional expense? Is the monitor relatively easy to use?

The answers to these questions now appear to be yes. In the current issue of this journal Dr. Manninen reviewed one centre's experience with SSEP monitoring during spinal surgery over 18 months. The results are 
similar to the experience reported by other institutions confirming the ability of SSEP to monitor the spinal cord effectively during spinal surgery and thus potentially to decrease the incidence of neurological injury. ${ }^{1,3-7}$ The author provides a good explanation of the aetiology of the high incidence of false positives (4.4\%) from their results. Of particular note is the false positive rate which has plagued the use of SSEP with rates reported from other studies being $0.5-20.0 \%$. The sensitivity of SSEP during spinal surgery varies from 87.5 to $100 \%$. The specificity is usually fairly broad in the 35-90\%+ range. However, almost all SSEP clinical studies have reported relatively high false positive rates and, as a result, the positive predictive value suffers. The negative predictive value is consistently high due in part to the low incidence of neurological injury. ${ }^{7}$

It is unfortunate that there are no controlled, randomized, double blind studies to show definitively the actual decrease in neurological injury when SSEPs are used but it is very difficult, given the available evidence, to stand by and watch a significant change in the SSEP and do nothing. The majority of large studies to date are surveys and retrospective reviews with or without historical controls.

What is the case against the use of SSEP? The most frequently quoted argument is that since the typical SSEP urilizes the posterior columns as their major ascending pathway an isolated ventral cord injury may go undetected while the SSEP remains intact. We commonly use monitoring modalities that are not perfect. For example, the ECG is a routine monitor in all our patients yet the most commonly monitored leads do not survey all portions of the myocardium and, thus, an isolated injury may be missed. It makes little sense to abandon spinal cord monitoring when, in reality, a large portion of the cord is surveyed and the likelihood of a pure ventral cord injury is extremely rare.

The actual technique involved in monitoring SSEP during spinal surgery varies slightly between centres but general protocols are similar. They all involve stimulation of a peripheral nerve, most commonly the posterior tibial or median. For lumbar or thoracic surgery individually stimulated posterior tibial nerves appear to be the standard. However, popliteal and sacral nerves are also used. Cervical spine surgery often utilizes the median nerve elicited SSEP with or without posterior tibial stimulation. Recording electrodes, either transcutaneous or transdermal, are placed along the spinal pathway and on the scalp over the corresponding somatosensory cortex. The montage used for the scalp electrodes is based on the International 10-20 System in accordance with the individual institution's protocol.
Standards for monitoring exist and are modified only slightly to fit the equipment and needs of the institution. Recordings acquired represent various anatomical points along the pathway and, as a result, an injury anywhere along the pathway should cause an alteration in the waveform obtained. The waveforms result from the repetitive electrical stimuli whose responses are averaged and cleaned to provide waveforms, which are then analysed. The SSEP waveforms are recorded in terms of latency (milliseconds) and amplitude (microvolts). Baseline SSEPs are established early in the surgical procedure at what may be called a "steady anaesthetic state." These baselines are used for comparison during the procedure. However, if anaesthetic depth is altered, new baselines should be obtained. Before and immediately after any major surgical manoeuvre it is advisable to obtain a new set of SSEP.

There are many factors that affect SSEP monitoring and the quality of tracings obtained. Of real, but sometimes exaggerated concern are the effects of anaesthetic agents. Most anaesthetic agents can be used without impairing the ability to record SSEPs. The effects of the commonly used agents have been characterized. ${ }^{8,9}$ Total intravenous anaesthesia (TIVA) with propofol may provide superior conditions for SSEP monitoring than inhalational anaesthesia. ${ }^{10}$ It is of paramount importance that anaesthetists have a thorough knowledge of the effects of the agents that they are using on the SSEP, and the SSEP must be interpreted taking those effects into account. There are many other factors that affect SSEP including patient-physiological factors and extrinsic factors. The physiological factors such as patient size, age, pre-operative neurological status, temperature, blood pressure, $\mathrm{P}_{\mathrm{a}} \mathrm{O}_{2}, \mathrm{P}_{\mathrm{a}} \mathrm{CO}_{2}$ and haemodilution all effect SSEP." Many extrinsic factors may adversely affect SSEP. Electrical interference from common operating room equipment such as blood warmers, microscopes, electric OR tables, fluoroscopy equipment, and electrical cables crossing SSEP monitoring wires can be quite problematic. Cautery causes the most interference and, at times, the surgeon may be asked to refrain temporarily from its use. The evoked potential equipment used in most ORs was not originally designed for use in the electrically hostile OR environment. However, the newer monitors have been adapted to function more efficiently in this setting making SSEP monitoring more user friendly, the results more reliable and less susceptible to outside interference. The majority of these factors can be controlled or modified to allow effective SSEP monitoring.

Should a change in the SSEP occur, it must first be determined if something other than neural injury has 
occurred. This must happen promptly and, with experience, the team can accomplish this efficiently. When a change occurs, if it is due to neural compromise, it has likely occurred a few minutes before the change in SSEP has been noted. Thus, prompt intervention is required. What constitutes a significant change in SSEP differs among centres. A decrease in amplitude of $25-50 \%$ is the range reported in most literature with $50 \%$ being the most commonly used parameter. An increase in latency between $5 \%$ and $10 \%$ is the usual quoted range with large numbers using $10 \%$ as their level of significance. ${ }^{7}$ Interventions are somewhat limited however; manoeuvres such as raising blood pressure, increasing inspired oxygen, and raising haematocrit if appropriate. The surgeon may also decrease the distraction of the spine, or remove retractors or hardware that may have caused the problem. Some advocate the use of the wake-up test in this situation. ${ }^{7}$

Whom should we monitor? With the economics of medicine playing an increasing role in what is provided to patients it is important that resources are allocated where they are likely to serve the greatest benefit. The literature is consistent with the view that patients undergoing corrective procedures for scoliosis be monitored: the majority of members of the Scoliosis Research Society use SSEP monitoring during major spinal surgery. ${ }^{7}$ Dr.Manninen also noted that those having thoracic spinal surgery had the greatest incidence of intraoperative SSEP change.

There are several keys to successful monitoring and all are important for reliable, useful information to be obtained. The entire team must be well educated about using SSEP in the OR setting and, as experience grows, reliability of the monitoring will likely increase. ${ }^{7}$ There must be excellent communication among the anaesthetist, electrophysiology technician (or neurophysiologist), and the surgeon. Without communication, monitoring will prove to be frustrating and likely of little benefit to the patients.

As more centres use SSEP monitoring, experience will grow, technology will improve and some of the problems with SSEP may be resolved. The use of other neuromonitoring modalities alone or in conjunction with SSEP may also serve to improve intraoperative spinal cord monitoring. With ongoing research with SSEP and other evoked potentials such as cerebellar evoked potentials, ${ }^{12}$ more complete spinal cord monitoring may soon be available. SSEP monitoring equipment is not cheap and neither are the salaries of the technicians or neurophysiologists but if major neurologic injuries can be prevented using this technology the benefits will surely outweigh the cost.

\section{Les potentiels évoqués somatosensitifs durant la chirurgie du rachis}

Même si en tant qu'anesthésistes nous surveillons l'intégrité de plusieurs systèmes au moyen d'une myriade de moniteurs, nous ne surveillons habituellement pas le système nerveux. Lors de plusieurs types d'opération, le tissu nerveux est soumis à un risque considérable de lésion et pourtant il demeure non surveillé. La chirurgie du rachis, particulièrement celle avec instrumentation comme lors de correction de scoliose est un type d'opération fréquent qui impose des risques de lésion élevés au tissu nerveux. Les conséquences catastrophiques d'une lésion permanente de la moelle sont évidentes. Les complications neurologiques de la chirurgie du rachis ne sont pas rares, l'incidence étant d'approximativement 1,6\%" Par le passé, le seul test peropératoire relativement fiable permettant de s'assurer de l'intégrité de la moelle était le test de répeil de Stagnara. Ce test exigeait qu'on réveille le patient durant l'opération, en général au moment du risque majeur de lésion, et qu'on lui demande de bouger ses membres. Ceci ne se fait pas sans risque car un patient éveillé peut s'extuber ou arracher ses voies veineuses, et il y a un risque accru d'embolie gazeuse si le patient se met à respirer spontanément. Du point de vue du patient, il peut être affolant d'envisager être éveillé en plein milieu de sa chirurgie. Le test d'éveil n'est pas fiable à $100 \%$ et c'est pourquoi on a cherché un monitorage fiable, relativement non invasif qui nous permet de surveiller la moelle de façon sécuritaire tout en gardant le patient anesthésié durant toute la procédure: c'est ce qui nous a conduits à utiliser les potentiels évoqués somatosensoriels ou somesthésiques (SSEP).

Les potentiels évoqués somesthésiques sont une représentation des réponses électrophysiologiques à la stimulation d'une voie afférente telles que mesurées au niveau du cortex somatosensitif. Ils reflètent le résultat net de l'activité neuronale des nerfs périphériques via la moelle jusqu'au cerveau. Le mérite scientifique et l'utilité clinique peropératoire des SSEP ont fait le sujet de débats depuis l'introduction de cette technique en salle d'opération il y a de ça plus de 20 ans $^{1}$. L'utilisation peropératoire des potentiels évoqués semble augmenter en Amérique du Nord, stimulée en partie par la prise de 
position écrite de la Scoliosis Research Society en 1992 qui recommande l'utilisation routinière des SSEP durant la chirurgie de la scoliose pour prévenir les lésions de la moelle ${ }^{2}$. Que ce soit le climat médico-légal qui prévaut actuellement ou la croyance que l'utilisation des SSEP apportera un bénéfice réel à nos patients subissant une chirurgie du rachis, les SSEPs vont probablement devenir une composante usuelle du monitorage en SOP dans un futur prévisible.

L'utilisation peropératoire des potentiels évoqués somesthésiques n'est pas limitée à la chirurgie du rachis puisque ce monitorage est aussi utilisé comme monitorage neurologique dans la chirurgie de la carotide, dans la chirurgie neurovasculaire, acétabulaire et dans la chirurgie nécessitant un clampage aortique. Bien que la surveillance des SSEP puisse aussi être utile durant les procédures vasculaires intra et extracrâniennes, les procédures acétabulaires et celles nécessitant un clampage aorrique, cette discussion sera restreinte à l'utilisation des potentiels évoqués somatoesthésiques durant la chirurgie du rachis.

Il faut répondre à plusieurs questions importantes. Les potentiels évoqués somatoesthésiques peuvent-ils être enregistrés de façon fiable en SOP sans faire courir au patient un risque accru de morbidité directement lié au monitorage? Est-ce que les changements peropératoires des potentiels somatoesthésiques correspondent à des déficits neurologiques postopératoires? Peut-on utiliser ces changements pour prévenir la survenue de déficits neurologiques? Les bénéfices valent-ils les coûts additionnels? Le moniteur est-il relativement facile à utiliser?

La réponse à ces questions semble maintenant être oui. Dans ce numéro, le Dr Manninen a passé en revue l'expérience d'un milieu avec le monitorage des potentiels évoqués somatoesthésiques durant une période de 18 mois. Les résultats sont analogues à ceux rapportés par d'autres centres, confirmant la capacité des SSEP à surveiller efficacement la moelle durant la chirurgie du rachis et donc à pouvoir diminuer l'incidence de lésions neurologiques ${ }^{1,3-7}$. L'auteur nous fournit aussi une bonne explication concernant l'étiologie de l'incidence élevée de faux positifs $(4,4 \%)$ dans son étude. Il faut aussi souligner le taux élevé de faux positifs qui a toujours ennuyé l'utilisation des SSEP, ce taux allant selon les études de 0,5 à $20 \%$. La sensibilité des SSEP durant la chirurgie du rachis varie de 87,5 à $100 \%$. La spécificité est en général assez large, dans un registre de 35 à $90+\%$. Cependant, presque toutes les études cliniques concernant les SSEP ont rapporté un taux de faux positifs relativement élevé avec, comme résultat, une baisse de la valeur prédictive positive. La valeur prédictive néga- tive demeure constamment élevée, en partie à cause de la faible incidence des lésions neurologiques?

Il est dommage qu'il n'y ait pas d'études contrôlées, aléatoires, à double insu pour montrer de façon définitive qu'il y a une diminution des lésions neurologiques lorsqu'on utilise les SSEP mais il est très difficile, face à l'évidence disponible, d'être présent et de regarder sans intervenir un changement significatif d'un potentiel somatoesthésique. Jusqu'à maintenant, la majorité des grandes études sont des enquêtes et des revues rétrospectives avec ou sans contrôles historiques.

Pourquoi refuser d'utiliser les potentiels somatoesthésiques? L'argument le plus fréquemment invoqué est que les potentiels somatoesthésiques typiques utilisent les colonnes postérieures comme trajet ascendant majeur et qu'une lésion de la moelle du côté ventral pourrait passer inaperçue, les SSEP demeurant inchangés. Pourtant, nous utilisons très fréquemment des modalités de monitorage qui ne sont pas parfaites. Par exemple, nous utilisons le monitorage de l'ECG chez tous nos patients mais les dérivations utilisées le plus fréquemment ne surveillent pas toutes les portions du myocarde, de sorte qu'une lésion localisée pourrait passer inaperçue. Il est donc peu logique d'abandonner le monitorage de la moelle quand, en fait, nous surveillons une grande partie de la moelle et que les chances d'une lésion isolée à la partie ventrale de la moelle sont extrêmement rares.

La technique utilisée dans la surveillance des SSEP durant la chirurgie du rachis varie légèrement d'un centre à l'autre mais les protocoles généraux sont semblables. Ils comprennent tous la stimulation d'un nerf périphérique, la plupart du temps le nerf tibial postérieur pour le membre inférieur ou le médian pour le membre supérieur. Pour les chirurgies lombaires ou thoraciques, la stimulation individuelle des nerfs tibiaux postérieurs semble être la norme. Cependant, les nerfs poplitée et sacré sont aussi utilisés. Pour la chirurgie de la colonne cervicale, on utilise souvent les potentiels provoqués au niveau du médian avec ou sans stimulation du tibial postérieur. Des électrodes pour enregistrer, transcutanées ou transdermiques, sont disposées près du trajet spinal, ainsi que sur la tête au-dessus du cortex somatosensitif correspondant. La disposition utilisée pour les électrodes du cuir chevelu est basée sur le système international $10-20$ et se conforme au protocole utilisé dans chaque centre. Les standards de monitorage existent et ne sont modifiés que légèrement pour s'adapter à l'équipement et aux besoins de chaque centre. Les enregistrements obtenus représentent différents points anatomiques le long d'un trajet et, comme résultat, une lésion n'importe où le long du trajet devrait causer une modification de l'onde obtenue. Les ondes 
sont le résultat de stimulations électriques répétitives dont on a fait la moyenne des réponses, tout en éliminant les artéfacts, pour ensuite les analyser. Les ondes des potentiels évoqués somatoesthésiques sont enregistrées en termes de temps de latence (millisecondes) et d'amplitude (microvolts). Les potentiels évoqués somatoesthésiques de base sont déterminés tôt dans le déroulement de l'opération à un moment que l'on pourrait appeler "état anesthésique stable ". Ces potentiels de base servent de référence durant l'intervention. Cependant, si la profondeur de l'anesthésie est modifiée, une nouvelle ligne de base doit être obtenue. Avant et après une manoeuvre chirurgicale majeure il est recommandé d'obtenir de nouveaux potentiels somatoesthésiques.

De nombreux facteurs affectent le monitorage des SSEP et la qualité des enregistrements obtenus. Les effets des agents anesthésiques représentent une préoccupation réelle mais parfois exagérée. La plupart des agents anesthésiques peuvent être utilisés sans modifier la capacité d'enregistrement des potentiels évoqués somatoesthésiques. Les effets des agents utilisés fréquemment ont été bien documentés ${ }^{8,9}$. L'anesthésie intraveineuse totale (TIVA) à base de propofol peut fournir de meilleures conditions pour le monitoring des SSEP que l'anesthésie d'inhalation ${ }^{10}$. Il est de la plus grande importance que les anesthésistes aient une connaissance approfondie des effets des agents qu'ils utilisent sur les SSEP, et les SSEP doivent être interprétés en tenant compte de ces effets. Il y a plusieurs autres facteurs qui modifient les SSEP que ce soit des facteurs liés à la physiologie du patient ou des facteurs extrinsèques. Les facteurs physiologiques tels la taille, l'âge, l'état neurologique préopératoire, la température, la pression artérielle, la $\mathrm{PaO}_{2}$, la $\mathrm{PaCO}_{2}$ et l'hémodilution affectent tous les potentiels évoqués somatoesthésiques ${ }^{11}$. Les SSEP sont aussi perturbés par plusieurs facteurs extrinsèques: l'interférence électrique provenant de l'équipement usuel de la SOP comme les réchauffes-sang, les microscopes, les tables d'opération électriques, l'équipement de fluoroscopie et les fils électriques croisant les fils de monitorage des SSEP, peut être problématique. Le cautère est l'appareil qui cause le plus d'interférence et, à certains moments, on demandera au chirurgien d'éviter temporairement de s'en servir. L'équipement utilisé dans la plupart des SOP pour l'enregistrement des potentiels évoqués n'a jamais été conçu pour une utilisation dans un environnement électriquement hostile comme celui de la salle d'opération. Cependant, les nouveaux moniteurs ont été modifiés de façon à fonctionner plus efficacement dans cet environnement rendant ainsi le monitorage des SSEP plus convivial, les résultats plus fiables et moins sujets aux interférences externes. La majorité des facteurs dont nous avons parlé peuvent être contrôlés ou modifiés pour permettre un monitorage efficace des SSEP.

Lorsqu'un changement au niveau des SSEP survient, il faut d'abord déterminer si quelque chose d'autre qu'un dommage neurologique est survenu. Ceci doit être réalisé rapidement et, avec l'expérience, l'équipe peut réussir ceci efficacement. Quand un changement survient, s'il est imputable à un dommage neurologique, ce dernier a dû survenir quelques minutes avant que le changement des SSEP soit noté. C'est pourquoi une intervention rapide est nécessaire. Ce qui constitue un changement significatif des SSEP varie selon les centres. Une diminution d'amplitude de 25-50\% est le registre rapporté dans la littérature, $50 \%$ étant la valeur la plus utilisée. Au niveau de la latence, une augmentation de 5 à $10 \%$ est le registre le plus souvent cité, la majorité utilisant $10 \%$ comme niveau significatif?. Lorsque ces changements surviennent, les interventions correctrices sont tout de même relativement limitées: on réalise les manoeuvres suivantes soit augmenter la pression artérielle, augmenter la $\mathrm{FiO}_{2}$ et augmenter l'hématocrite si cette mesure est approprié; le chirurgien peut diminuer la distraction de la colonne ou enlever les rétracteurs ou la quincaillerie qui peuvent avoir causé le problème. Certains préconisent de faire un test de réveil dans cette situation?

Qui devons-nous monitorer? Dans le contexte où les aspects économiques de la médecine jouent un rôle croissant dans la détermination de ce qui est offert aux patients, il est important d'allouer les ressources aux endroits où le bénéfice risque d'être le plus grand. La littérature est unanime à recommander un monitorage pour la chirurgie correctrice de la scoliose: la majorité des membres de la Scoliosis Research Society utilisent le monitorage des SSEP durant la chirurgie du rachis ${ }^{7}$. Le Dr Manninen a aussi noté que les patients opérés du rachis thoracique avaient l'incidence la plus élevée de modifications peropératoires des SSEP.

Il y a plusieurs secrets pour réaliser un monitorage réussi et tous sont importants si on désire obtenir une information utile et fiable. Toute l'équipe doit être bien formée à l'utilisation des SSEP dans le contexte de la SOP et, à mesure que l'expérience s'accumule, la fiabilité du monitorage va s'accroître? ${ }^{7}$. Il doit exister une excellente communication entre l'anesthésiste, le technicien en électrophysiologie (ou le neurophysiologiste) et le chirurgien. Sans communication, le monitorage va devenir source de frustration et apportera peu de bénéfice aux patients.

À mesure que le nombre de centres utilisant le monitorage des SSEP s'accroît, la technologie va s'améliorer et quelques uns des problèmes vont se résoudre. 
L'utilisation d'autres modalités de monitorage neurologique seules ou en association avec les SSEP serviront à améliorer la surveillance de la moelle. Avec toute la recherche concernant les SSEP et les autres potentiels évoqués tels que les potentiels évoqués cérébelleux ${ }^{12}$, un monitorage plus complet de la moelle sera peut être bientôt disponible. L'équipement de monitorage des potentiels évoqués somatoesthésiques n'est pas bon marché, pas plus que les salaires des techniciens ou des neurophysiologistes, mais si l'utilisation de cette technologie permet d'éviter des dommages neurologiques majeurs, les bénéfices dépassent sûrement les coûts.

\section{References}

1 Kalkman CJ, Been HA, Ongerboer de Visser BW. Intraoperativc monitoring of spinal cord function. A review. Acta Orthop Scand 1993; 64: 114-23.

2 Scoliosis Research Society. Somatosensory evoked potential monitoring of neurologic cord function during spinal surgery. A position paper. Park Ridge, Il: Scoliosis Research Society, 1992.

3 Forbes HJ, Allen PW, Waller CS, Jones SJ, Edgar MA, Webb PJ. Spinal cord monitoring in scoliosis surgery. Experience with 1168 cases. J Bone Joint Surgery $\mathrm{Br}$ 1991; 73: 487-91.

4 Dawson EG, Sherman JE, Kanim LEA, Nuwer MR. Spinal cord monitoring. Results of the Scoliosis Research Society and the European Spinal Deformity Society survey. Spine 1991; 16: S361-4.

5 Grundy $B L$. Intraoperative monitoring of sensoryevoked potentials. Anesthesiology 1983; 58: 72-87.

6 Dinner DS, Luders H, Lesser RP, Morris HH, Barnett G, Klem G. Intraoperative spinal sometosensory evoked potential monitoring. J Neurosurg 1986; 65: 807-14.

7 Nuwer MR, Dawson EG, Carlson LG, Kanim LEA, Sherman JE. Somatosensory evoked potential spinal cord monitoring reduces neurologic deficits after scoliosis surgery: results of a large multicenter survey. Electroencelaphalogr Clin Neurophysiol 1995; 96: 6-11.

8 Peterson DO, Drummond JC, Todd MM. Effects of halothane, enflurane, isoflurane, and nitrous oxide on somatosensory evoked potentials in humans. Anesthesiology 1986; 65: 35-40.

9 Lam AM, Sharar SR, Mayberg TS, Eng CC. Isoflurane compared with nitrous oxide anaesthesia for intraoperative monitoring of somatosensory-evoked potentials. Can J Anaesth 1994; 41: 295-300.

10 Taniguchi $M$, Nadstawek J, Pechstein U, Schramm J. Total intravenous anesthesia for improvement of intraoperative monitoring of somatosensory evoked potentials during aneurysm surgery. Neurosurgery 1992; 31 : 891-7.
11 Goodrich JT. Electrophysiologic measurements: intraoperative evoked potential monitoring. Anesthesiology Clinics of North America 1987; 5: 477-87.

12 Hurlbert RJ, Feblings MG, Moncada MT. Use of sensoryevoked potentials recorded from the human occiput for intraoperative physiologic monitoring of the spinal cord. Spine 1995; 20: 2318-27. 